Basic Science

\title{
e Experimental Evaluation of Percutaneous Lumbar Laser Disc Decompression Using a 1414 nm Nd:YAG Laser
}

Bong Ju Moon, MD, Hye Yeong Lee, MS, Keung Nyun Kim, MD, PhD, Seong Yi, MD, PhD, Yoon Ha, MD, PhD, Do Heum Yoon, MD, PhD, and Dong Ah Shin, MD, PhD

From: Yonsei University College of Medicine, Seoul,

Korea

Address Correspondence: Dong Ah Shin, MD, PhD

Dept of Neurosurgery Yonsei University College of Medicine

50, Yonsei-ro, Seodaemun-gu Seoul, Korea

E-mail:shindongah@ me.com

Conflict of interest and Funding information on P.

1098.

Manuscript received: 03-19-2015 Revised manuscript received: 06-07-2015

Accepted for publication: 06-17-2015

Free full manuscript: www.painphysicianjournal.
Background: Laser ablation under an epiduroscopic view allows for the vaporization of a small amount of the nucleus pulposus, causing a reduction in intradiscal pressure and relief of radicular pain Currently, Ho:YAG and Nd:YAG lasers are commonly used for spinal diseases. However, the use of the $\mathrm{Nd}$ :YAG laser for intra-spinal procedures can be limited because of thermal injury and low efficacy.

Objective: To investigate the efficacy and safety of epiduroscopic laser ablation using a $1414 \mathrm{~nm}$ $\mathrm{Nd}$ :YAG laser, we examined that laser ablation was able to penetrate nucleus pulposus without heating surrounding tissues and without mechanical damage to surrounding tissue.

Study Design: Our experiment involved live and cadaveric animal studies and a human cadaveric study.

Setting: University in Korea.

Methods: Two live pigs, 3 porcine cadavers, and 2 human cadavers were used. For the in vitro study, intradiscal and epidural pressure and temperature were compared in vertebral columns obtained from 3 porcine cadavers before and after laser ablation. For the in vivo study, 2 pigs were used to simulate percutaneous epiduroscopic laser ablation. They were observed for behavioral changes and neurological deficits for one month after the laser ablation procedure. Two human cadavers were used for placing the laser fiber and epiduroscope in the correct target site through the sacral hiatus. Histological analysis was also performed to observe any damage around the ablated lesion.

Results: Both intradiscal and epidural pressure were markedly reduced immediately after laser ablation as compared with the pre-ablative state. The amount of the pressure decrease in the intradiscal space was significantly greater than that in the epidural space ( $45.8 \pm 15.0$ psi vs. $30.0 \pm 9.6$ psi, $P=0.000)$. The temperature beneath the ipsilateral spinal nerve, which was the nearest site to the laser probe, never exceeded $40^{\circ} \mathrm{C}$. Histology revealed no evidence of thermal damage to surrounding structures, including the spinal nerves, end plates, and vertebrae, after laser ablation. All live pigs showed normal behavior without any sign of pain. In the human cadaveric study, there was no case of targeting failure or dural laceration. The mean time to reach the target region was less than 5 minutes.

Limitations: The pressure measurements were performed on cadavers and not in vivo. Cadaver models cannot account for intradiscal pressure changes that occur during live muscle contraction and different positions, which may affect results. Moreover, although we controlled temperatures with heat baths, vascular and cerebrospinal fluid circulations were not simulated. Those circulations may change the temperature results in vivo.

Conclusions: The $1414 \mathrm{~nm}$ Nd:YAG laser can be used effectively and safely under the guidance of a spinal epiduroscope in an in vivo porcine model and in a human cadaveric model.

Study Approval: Approval for the current study was granted by the Institutional Review Board of our institute (approval number: 1-2014-0049).

Key words: Intervertebral disc disease, laser-assisted spinal endoscopy, disc decompression, porcine spine, cadaver, animal model, intradiscal pressure, epidural pressure, Nd:YAG laser, laser ablation

Pain Physician 2015; 18:E1091-E1099 
aser ablation under an epiduroscopic view can be an effective method for treating intraspinal pathologies, such as a herniated nucleus pulposus and painful microscopic adhesions. The level of evidence is II-1 or II-2 for short- and long-term relief for endoscopic adhesiolysis in post-lumbar surgery syndrome, based on one randomized trial (1). Although the epiduroscopic procedure has long been used and the accumulated evidence supports its beneficial effect, the procedure's ability to remove pathologic adhesions has been under debate until recently. Its inability to remove pathology which limits its use in epidural adhesiolysis and results in less favorable outcomes, may be overcome by laser ablation. Laser ablation may be preferable to open surgery because it requires no dissection, results in less bleeding, causes less tissue damage, and has a smaller risk of re-fibrosis (2).

Currently, Ho:YAG is the most commonly used laser for ablation therapy. The Ho:YAG laser has been accepted as the safest laser because of low tissue penetration. The Nd:YAG laser, which was introduced earlier than was the Ho:YAG laser, was not used for deep tissue penetration. However, the Nd:YAG laser can also provide shallow tissue penetration by adjusting its wavelength to that of the Ho:YAG laser (3). The advantages of the $\mathrm{Nd}$ :YAG include easier laser control, a smaller manufacturing fee, and equivocal effectiveness $(4,5)$. To the best of our knowledge, there has been no report on the use of a $1414 \mathrm{~nm} \mathrm{Nd:YAG} \mathrm{laser} \mathrm{for} \mathrm{intraspinal} \mathrm{use.} \mathrm{The} \mathrm{pur-}$ pose of this study was to investigate the efficacy and safety of epiduroscopic laser ablation with the $1414 \mathrm{~nm}$ $\mathrm{Nd}$ YAG laser.

\section{Methods}

\section{Research Design}

Approval for the current study was granted by the Institutional Review Board of our institute (approval number: 1-2014-0049). In this study, 2 live pigs, 3 porcine cadavers, and 2 human cadavers were used.

For the in vitro study, whole vertebral columns were obtained from 3 porcine cadavers and tested within 2 hours of necropsy. All muscular structures were removed with caution so as not to damage the intervertebral discs. The lumbar spinal column was obtained from one specimen for temperature measurement. For pressure measurement, a total of 24 intervertebral discs from 2 fresh porcine cadavers were examined. Each specimen was wrapped in soft vinyl to prevent drying during the experiment. The specimens were divided into 2 groups of 12 intervertebral discs each and numbered alternatively as follows: Group 1, intradiscal ablation, $60 \mathrm{~J}$; Group 2, epidural ablation, $60 \mathrm{~J}$. Two live pigs were used for behavioral observation following percutaneous epidural laser ablation. Operations were also simulated in 2 human cadavers. For laser irradiation, an Nd:YAG laser (Lutronics, Ilsan, South Korea) with a wavelength of $1414 \mathrm{~nm}$ operating in the $0.75-12 \mathrm{~W}$ range was used. The laser energy was delivered through a $550 \mu \mathrm{m}$ bare optical fiber. The spot size on the tissue surface was $1.2 \mathrm{~mm}$ using the focusing lens, and the power applied ranged from 0.75 to $12 \mathrm{~W}$, corresponding to a power density of 3.16 $50.53 \mathrm{~W} / \mathrm{mm} 2$. Using the bare fiber tip, energy levels of the Nd:YAG laser ranged from 10 to $12 \mathrm{~W}$ delivered as pulses one or 2 seconds in duration.

\section{Temperature Measurement}

A fresh porcine cadaveric spine was used for the thermal analysis. The specimen consisted of 2 lumbar vertebral bodies and one intervertebral disc. The paravertebral muscles were removed. In each trial, 5 thermometer needles were placed into the spinal canal, as follows: Sensor 1, underneath the ipsilateral spinal nerve; Sensor 2, ipsilateral epidural space; Sensor 3, intradural space; Sensor 4, contralateral epidural space; and Sensor 5, underneath the contralateral spinal nerve (Fig. 1). The position was confirmed using orthogonal radiographs in the anterior-posterior and lateral views by a fluoroscope (BV Pulsera; Philips, Amsterdam, the Netherlands). The spine installed with temperature sensors was placed within a temperature-controlled saline bath and allowed to reach thermal equilibrium (Fig. 2). The water bath was $5 \mathrm{~L}$ in size with a constant, controlled temperature of $37^{\circ} \mathrm{C} \pm 1^{\circ} \mathrm{C}$. Saline was circulated with a $300-r p m$, continuously rotating magnetic stir bar. The flow rate within the bath was maintained at a low level to avoid excessive convective cooling at the outer boundary of tissue but still maintain adequate temperature control of the interface. The laser probe was placed into the target area. Temperature was continuously recorded along with laser ablation (10, 20, 30, 40, 50, $60 \mathrm{~J}$ for 10 seconds) using a thermometer (TC-08; OMEGA Engineering, Stamford, CT, USA). The temperature was registered with an accuracy of $0.01^{\circ} \mathrm{C}$ and a frequency of $0.5 \mathrm{~Hz}$ over 800 single measurements per thermocouple. Each test lasted 5 minutes. The timetemperature curve was obtained and analyzed. 

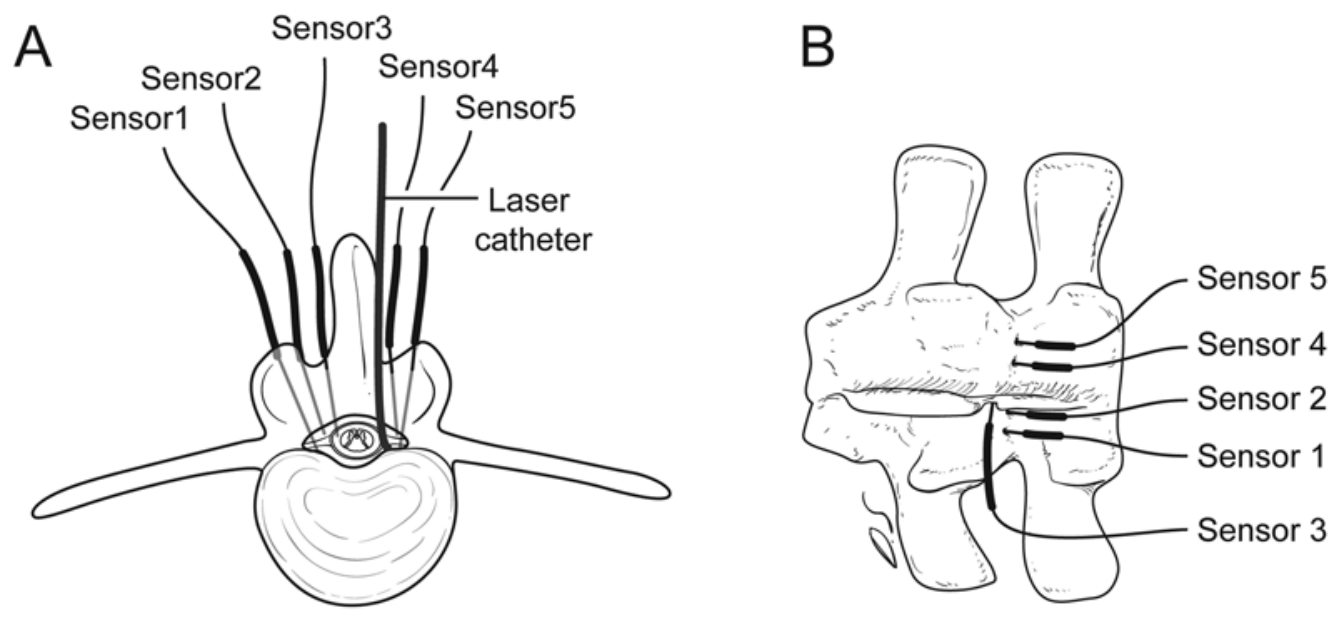

Fig. 1. Five thermometer needles placed into the spinal canal from a porcine cadaver: Sensor 1, underneath the ipsilateral spinal nerve; Sensor 2, ipsilateral epidural space; Sensor 3, intradural space; Sensor 4, contralateral epidural space; Sensor 5, underneath the contralateral spinal nerve.

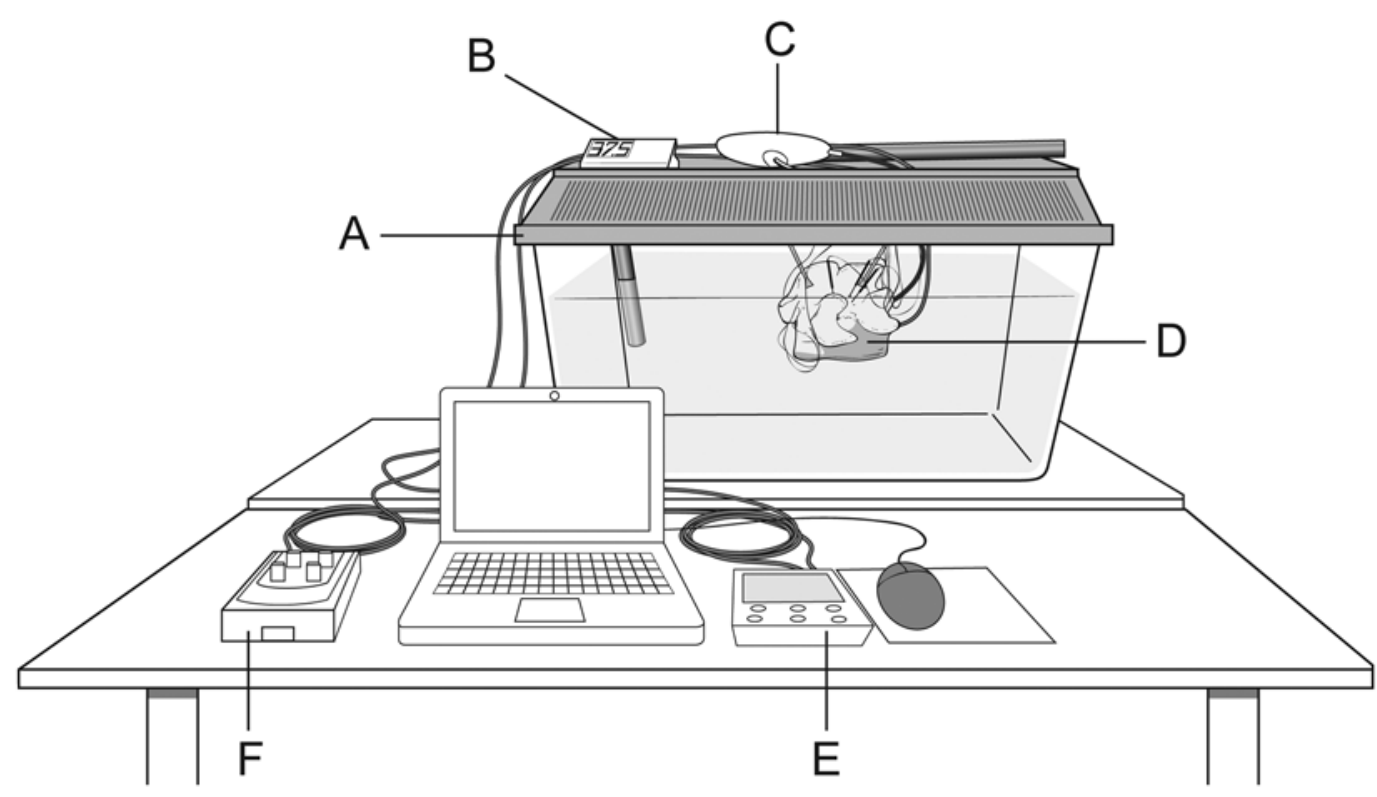

Fig. 2. Experimental setting: The porcine spine installed with temperature sensors was placed within a temperature-controlled saline bath and allowed to reach thermal equilibrium. A: saline bath, B: controller of temperature, C: laser catheter, D: porcine cadaveric spinal segment, E: thermometer, F: thermocouple 


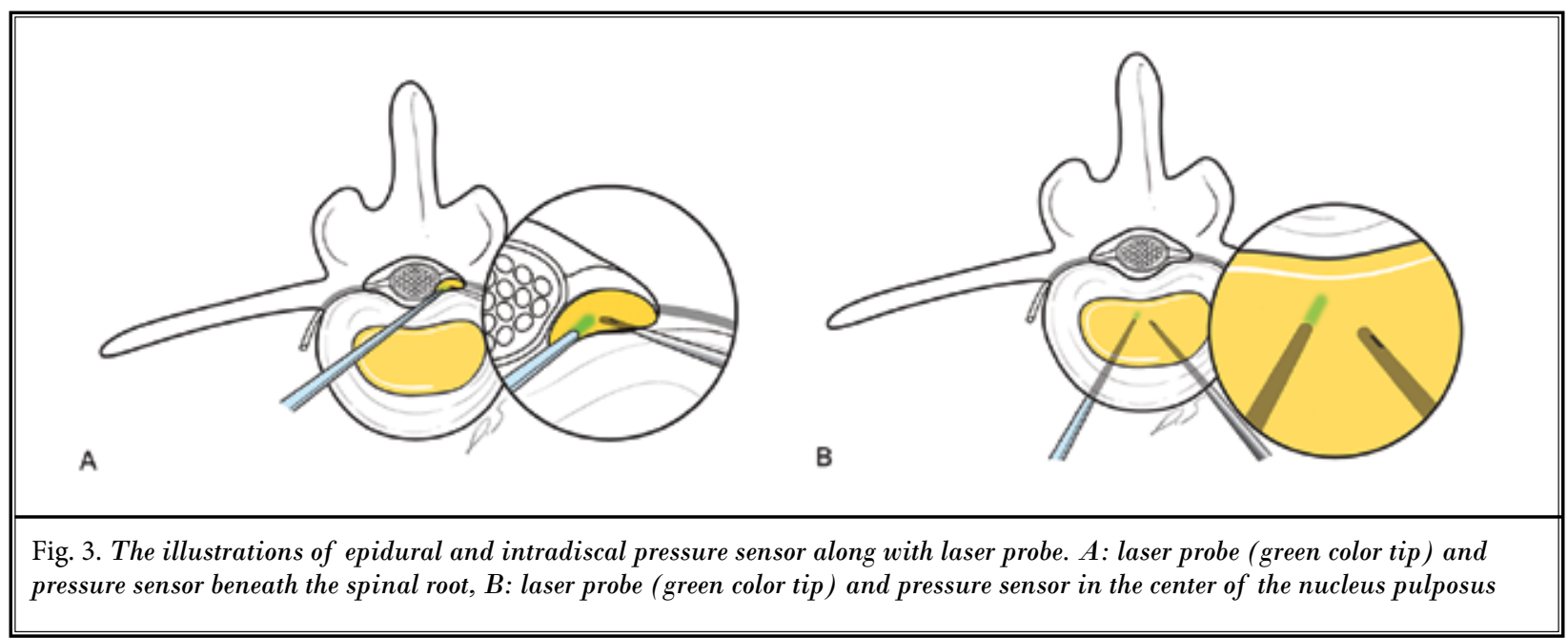

\section{Pressure Measurement}

For the measurement of pressure, a pressure sensor was connected to a computer-controlled data acquisition unit (Gaeltec S7d, Gaeltec, Isle of Skye, Scotland). The epidural pressure (EDP) measured underneath the ipsilateral nerve root was elevated after plugging the epidural space with the nucleus pulposus (Fig. 3A). For EDP measurement, a pressure sensor was introduced beneath the spinal root, which was compressed by the placed nucleus pulposus. For intradiscal pressure (IDP) measurement, a pressure sensor was introduced into the center of the nucleus pulposus of each disc with a posterolateral approach (Fig. 3B). The position was confirmed by the fluoroscope. The pressure was continuously recorded along with laser ablation using the laser device. The pressure was registered with an accuracy of $0.01 \mathrm{psi}$ and a frequency of $0.5 \mathrm{~Hz}$ over 800 single measurements per thermocouple. Each test lasted 5 minutes. The time-pressure curve was obtained and analyzed.

\section{In Vivo Study}

Two pigs, 5 months old and weighting $50 \mathrm{~kg}$, were used to simulate percutaneous epiduroscopic laser ablation. The pigs were sedated using intramuscular injection of ketamine $(20 \mathrm{mg} / \mathrm{kg})$. The pigs were placed on a radiolucent table, intubated, and ventilated on a respirator administering a mixture of oxygen (10\%) and air $(90 \%)$. Anesthesia was maintained by alpha chloralose (100 mg/kg bolus and $30 \mathrm{mg} / \mathrm{kg} / \mathrm{hour}$ ). An epidural, steerable, fiber-optic catheter system was introduced into the caudal region through the sacral hiatus $(2.7 \mathrm{~mm}$ Video Guided Catheter, Myelotec,
Rosewell, GA). A laser fiber and epiduroscope (1.3 mm Flexible Fiberoptic Endoscope, Myelotec, Rosewell, GA) were introduced through the steerable catheter. Using fluoroscopic and visual guidance, the apparatus was advanced toward the L5-S1 level. Epidurogram was performed with $5 \mathrm{~mL}$ of 180 concentration iohexol to check the ventral position of the catheter tip. We further steered the catheter toward the right side to work specifically on the annular surface. The laser was set at $0.5 \mathrm{~J}$ output at 5 pulses/second. We lased the region for 10 seconds without moving the catheter tip against the annular surface using epiduroscopic visualization. After the laser procedure, the pigs were continuously monitored until they were able to sit in sternal recumbency of their own accord. Then, the animals were returned to their home pen, and the behavior of individual pigs was recorded for one month. Observations on appearance of the incision, behavioral characteristics, eating, drinking, urination, defecation, temperature, pulse, and respiration were carefully analyzed.

\section{Cadaveric Study}

A human cadaver was placed in the prone position on a fluoroscopic table. Laser ablation was performed on a human cadaver using the same epidural, fluoroscopic, and epiduroscopic materials and procedures as noted above for the in vivo study in pigs. After completion of the procedure, a laminectomy of the L5 was performed with a midline skin incision. The L5-S1 ventral epidural space was exposed to confirm whether the catheter tip was placed correctly on the target site without damaging the adjacent nervous structures. 


\section{Statistical Analysis}

The results are expressed as the means \pm standard deviations. Student's t-test was used to assess statistical differences between groups using SAS software for Windows (SAS Institute Inc., Cary, NC). A $P<0.05$ was considered significant.

\section{Results}

\section{Temperature}

Temperature measurement was successfully performed in one porcine cadaver. The temperature increased immediately after laser ablation and reached the maximum temperature in 23 seconds, 54 seconds, 86 seconds, 108 seconds, and 181 seconds at the ipsilateral spinal nerve, ipsilateral epidural space, intradural space, contralateral epidural space, and contralateral spinal nerve, respectively. The temperature decreased to the preoperative state 472 seconds after stopping laser ablation. The maximum temperature at each site correlated with the distance from the ablation site. The temperature recorded at the ipsilateral spinal nerve, which was nearest to the laser probe, never exceeded $40^{\circ} \mathrm{C}$ (Fig. 4). The maximum temperature developed at the setting of $60 \mathrm{~J}$. The maximum temperature correlated with the distance from the ablation site and also did not exceed $40^{\circ} \mathrm{C}$ (Fig. 5). Thus, thermal injury as a consequence of laser ablation was very unlikely. The temperature may be lower in the live human body due to vascular circulation and its heat sink effect (6).

\section{Pressure}

Pressure measurement was successfully performed in 24 healthy intervertebral segments from the 2 porcine cadavers. Both IDP and EDP were markedly reduced immediately after laser ablation as compared with the preoperative state $(P=0.000)$. Preoperative IDP was $60.2 \pm 22.3$ psi, which decreased to $14.3 \pm 12.1$ psi after laser ablation $(P=0.000)$. Preoperative EDP was $34.6 \pm$ $9.7 \mathrm{psi}$, which decreased to $4.6 \pm 2.5 \mathrm{psi}$ after laser ablation $(P=0.000)$. In Fig. 6 , the amount of the pressure decrease in the intradiscal space was significantly larger than that in the epidural space $(45.8 \pm 15.0$ psi vs. 30.0 \pm 9.6 psi, $P=0.000$ ).

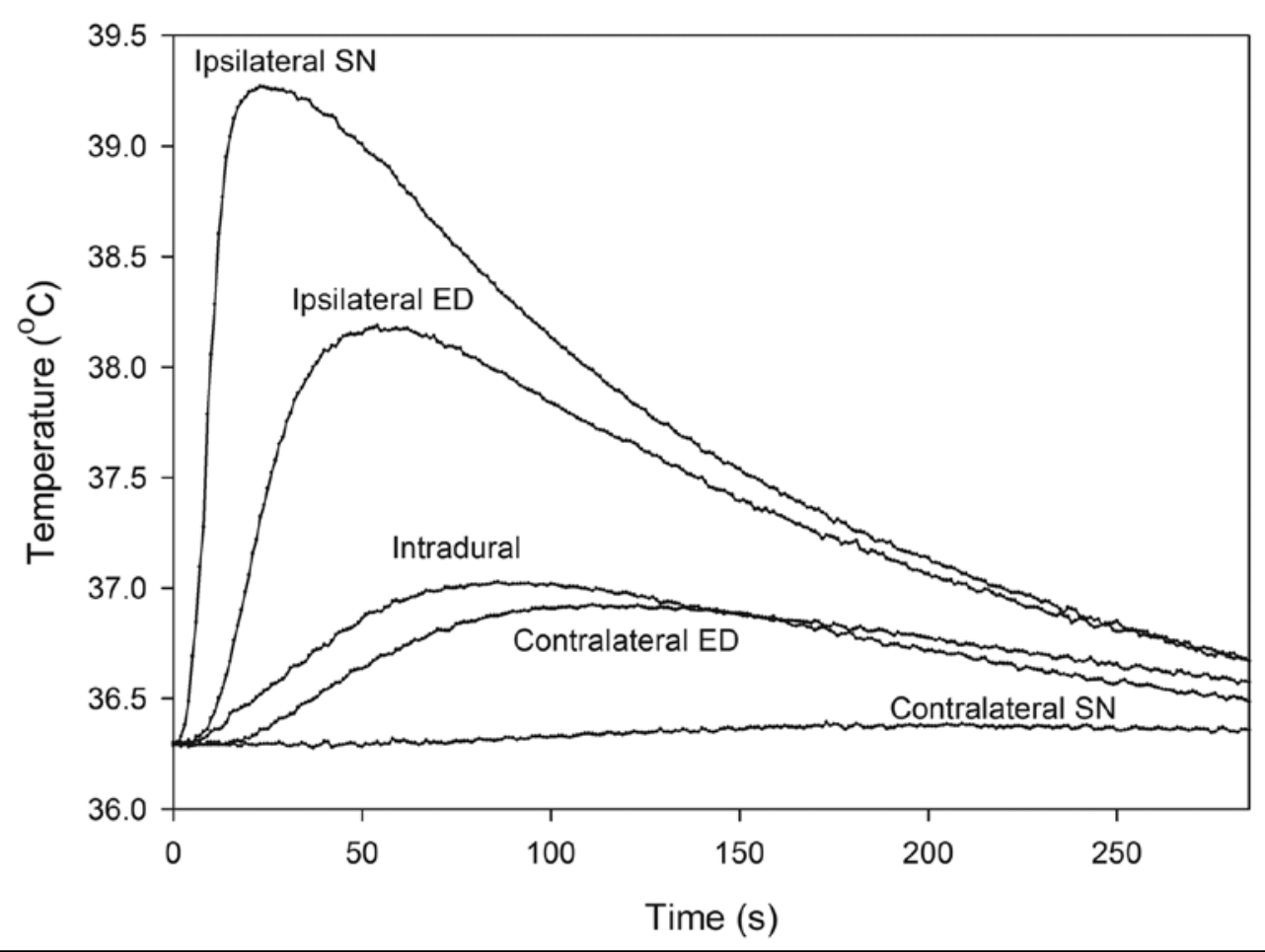

Fig. 4. The temperature increased immediately after laser ablation. The temperature decreased to the preoperative state 472 seconds after stopping laser ablation. SN: spinal nerve, ED: epidural space. 


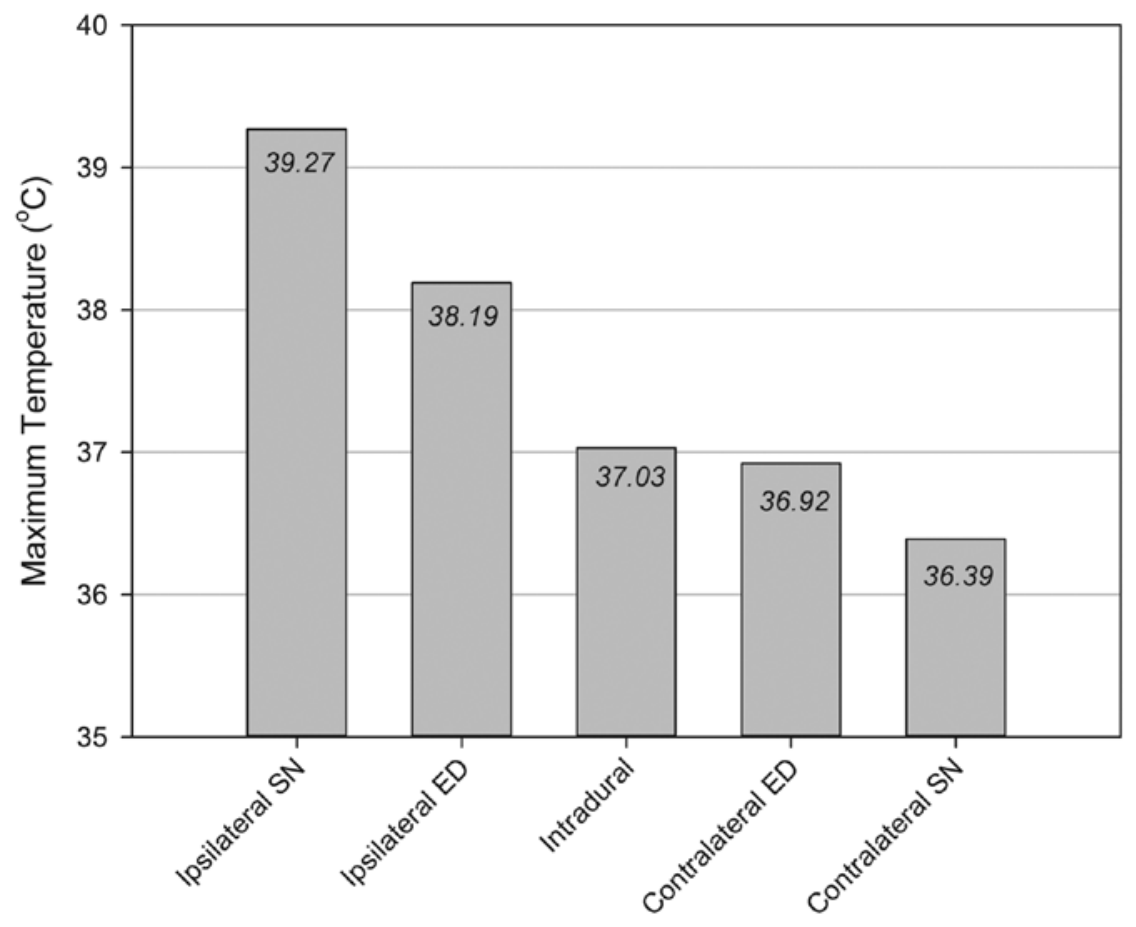

Fig. 5. The maximum temperature developed at the setting of $60 \mathrm{~J}$. The maximum temperature correlated with the distance from the ablation site and did not exceed $40^{\circ} \mathrm{C}$. SN: spinal nerve, ED: epidural space.

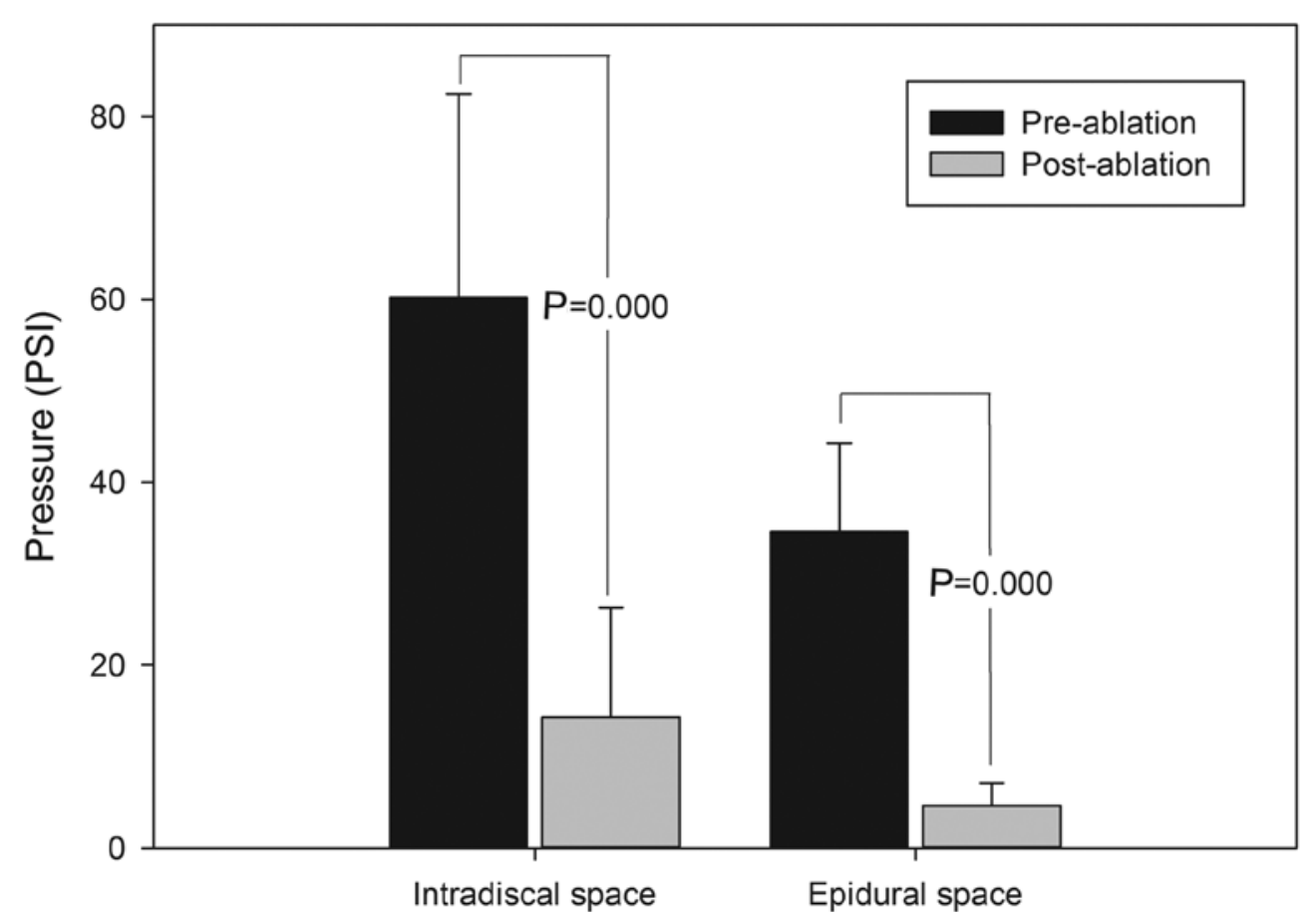

Fig. 6. The pressure measured in the intradiscal space and epidural space before and after laser ablation. The pressure was markedly reduced after laser ablation in both spaces. 


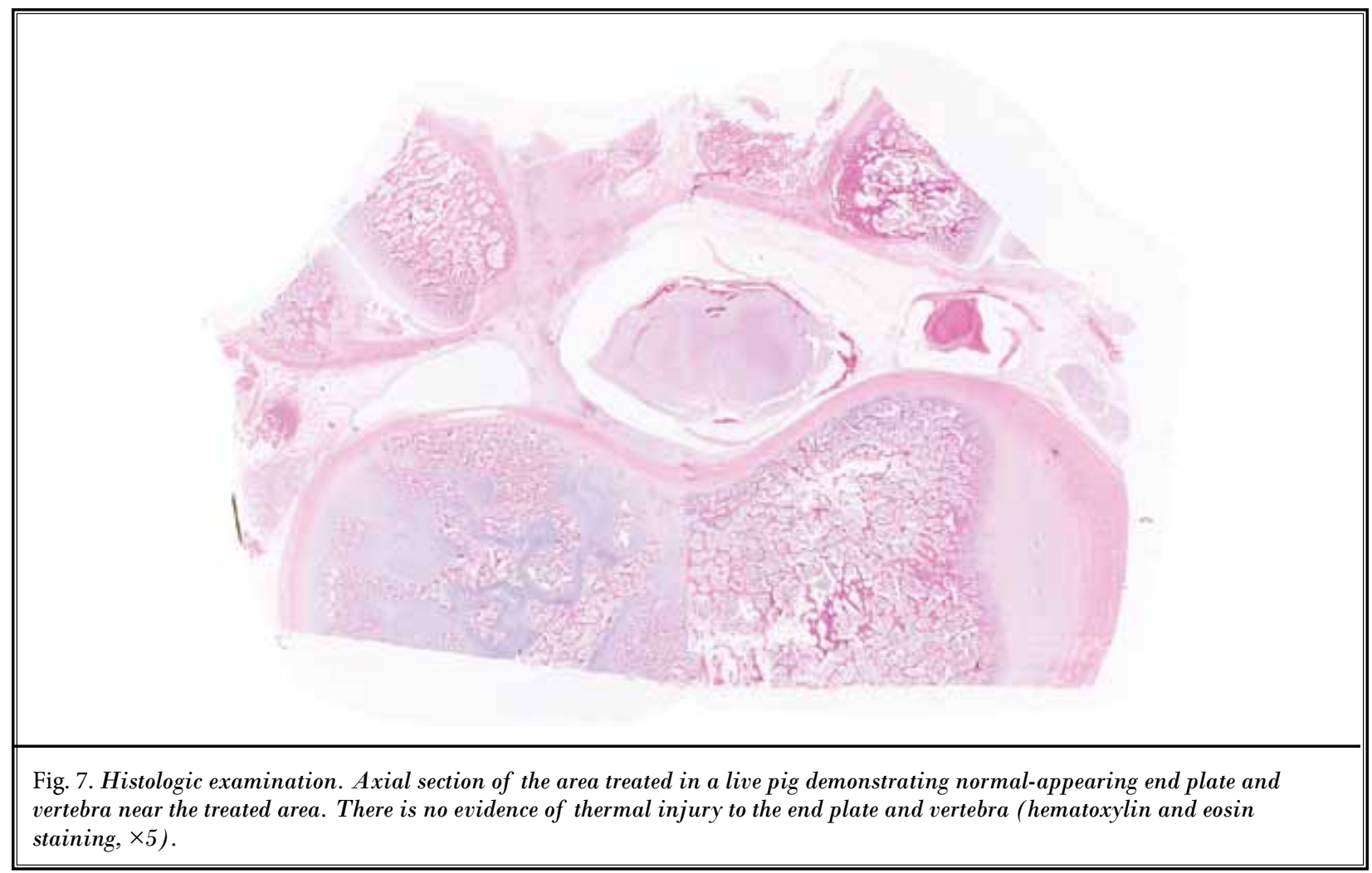

\section{In Vivo Experiment}

All pigs showed no abnormal behavior without any signs of pain. The time spent lying, nursing, sitting, standing, and walking did not differ between the preand postoperative periods.

\section{Histology}

Histologic slides of lumbar $4 / 5$ discs and endplates obtained from 2 live pigs one week after laser ablation were stained with hematoxylin and eosin (Fig. 7). In the axial sections, laser ablation formed oval cavities in the nucleus pulposus and round coagulations on the annulus fibrosus. However, tissue charring was not observed around the ablated lesion. There was no evidence of thermal damage to surrounding structures, including the spinal nerves, end plates, and vertebrae.

\section{Human Cadaveric Study}

A total of 5 catheterizations were attempted and successfully completed. There was no case of targeting failure or dural laceration. The mean time to reach the target region was less than 5 minutes.

\section{Discussion}

The purpose of our study was to investigate the efficacy and safety of epiduroscopy with Nd:YAG laser ablation to identify and remove target lesions in vivo porcine models and in human cadaveric models before the clinical application. The following questions were addressed: Can Nd:YAG laser ablation decrease intraspinal pressure by removing the nucleus pulposus effectively? Can Nd:YAG laser ablation be delivered epiduroscopically without thermal and mechanical injury to the surrounding structures?

Our study confirmed that a $1414 \mathrm{~nm}$ Nd:YAG laser significantly decreased IDP and EDP by ablating the nucleus pulposus. Pressure decreased by $76-87 \%$ immediately after $60 \mathrm{~J}$ 1414-nm Nd:YAG laser ablation. EDP also decreased significantly after laser ablation to the nucleus pulposus. While previous studies reported that $1000 \mathrm{~J}$ is necessary for decreasing IDP, we demonstrated that less energy can also significantly decrease pressure $(7,8)$, which may be because there some differences between the porcine and human spine. The porcine spinal canal and disc height are smaller than 
their human counterparts (9), and porcine discs also have a smaller disc volume and thinner posterior annulus than do human discs (10). Another reason for the smaller energy requirement may be due to the different decompression techniques used in the current study. EDP, measured underneath the ipsilateral nerve root, was elevated after plugging the epidural space with the nucleus pulposus. The elevated EDP decreased significantly after laser ablation to the nucleus pulposus and IDP also decreased, perhaps because the porcine nucleus pulposus was very small and gelatinous, and it may be easy to make a hole through the thinner posterior annulus by laser ablation. Although the energy of this laser ablation was insufficient to remove the entire nucleus pulposus, the volume reduction significantly decreased the intraspinal pressure due to the hydraulic phenomenon $(7,11,12)$. Furthermore, in the clinical setting, we hypothesize that macrophages can migrate into the herniated disc through the laser-perforated holes and desiccate the nucleus pulposus more rapidly $(13,14)$.

This study also demonstrated that $6 \mathrm{~W}$ delivery of $1414 \mathrm{~nm} \mathrm{Nd:YAG} \mathrm{laser} \mathrm{does} \mathrm{not} \mathrm{increase} \mathrm{temperature}$ to harmful levels. Furthermore, histology revealed no evidence of thermal damage to surrounding structures, including the spinal nerves, end plates, and vertebrae. Owing to the smaller amount of energy used and the low exothermic nature of the Nd:YAG laser, there was no significant temperature elevation around the ablated lesion, which should facilitate the use of the 1414-nm Nd:YAG laser for intraspinal use.

In the porcine cadaveric study, although we controlled the temperature with a heat bath, vascular and cerebrospinal fluid circulations were not simulated. Those circulations have been known to play a role in heat sink in vivo. Without the heat sink, this study showed no significant temperature elevation. Considering the additional heat sink from vascular and cerebrospinal fluid circulation, the Nd:YAG laser can be safely used in humans. Compared with the Nd:YAG laser, the Ho:YAG laser generates too much heat and must be cooled by saline irrigation (11). It has been suggested that percutaneous laser nucleolysis using the Ho:YAG laser can damage endplates from excessive thermal energy (15). Although compared with the Ho:YAG laser, the Nd:YAG laser provides deeper tissue penetration, its lower heat generation may make thermal injury less likely (4). By targeting the lesion accurately with the aid of an epiduroscope, the risk of thermal injury may further be minimized (16).

The limitation of this study was that pressure measurements were performed on cadavers and not in vivo. Cadaver models cannot account for IDP changes that occur during live muscle contraction and different postural positions (sitting, standing, etc.), which may affect results. While the current study only investigated decompression, the ability of the Nd:YAG laser to lyse adhesions may further improve spinal pain by the release of scar tissues (2). This hypothesis will be addressed in a future study.

\section{Conclusion}

The $1414 \mathrm{~nm} \mathrm{Nd:YAG} \mathrm{laser} \mathrm{is} \mathrm{effective} \mathrm{and} \mathrm{safe} \mathrm{for}$ decompressing herniated intervertebral discs under the guidance of a spinal epiduroscope in vivo porcine models and in human cadaveric models. Consequently, this study can be a foundation of clinical application of laser for human lumbar disc decompression.

\section{Acknowledgments}

\section{Funding/Support:}

1. This work was supported by the Industrial R\&D program of MOTIE/KEIT (Ministry of Trade, Industry and Energy/Korea Evaluation Institute of Industrial Technology). (10043086, Developement of 1,414nm Nd:YAG laser system for Minimal Invasive Spine Surgery)

2. This study was supported by a grant from the Korea Health technology R\&D Project, Ministry of Health \& Welfare, Republic of Korea (A120254).

This study received level 6 support from 2 former funding.

\section{Conflict of interest:}

Each author certifies that he or she, or a member of his or her immediate family, has no commercial association (i.e., consultancies, stock ownership, equity interest, patent/licensing arrangements, etc.) that might pose a conflict of interest in connection with the submitted manuscript. 


\section{References}

1. Manchikanti L, Boswell MV, Rivera JJ, Pampati VS, Damron KS, McManus CD, Brandon DE, Wilson SR. A randomized, controlled trial of spinal endoscopic adhesiolysis in chronic refractory low back and lower extremity pain. BMC Anesthesiology 2005; 5:10.

2. Epstein J, Adler R. Laser-assisted percutaneous endoscopic neurolysis. Pain Physician 2000; 3:43-45

3. Jayasree R, Gupta AK, Bodhey NK, Mohanty $M$. Effect of 980-nm diode laser and 1064-nm Nd:YAG laser on the intervertebral disc-in vitro and in vivo studies. Photomedicine and Laser Surgery 2009; 27:547-552.

4. Marguet CG, Sung JC, Springhart WP, L'esperance JO, Zhou S, Zhong P, Albala DM, Preminger GM. In vitro comparison of stone retropulsion and fragmentation of the frequency doubled, double pulse nd:yag laser and the holmium:yag laser. The Journal of Urology 2005; 173:1797-1800.

5. Choy DS, Altman P, Trokel SL. Efficiency of disc ablation with lasers of various wavelengths. Journal of Clinical Laser Medicine \& Surgery 1995; 13:153-156.

6. Mishan Y, Mosyak A, Pogrebnyak E,
Hetsroni G. Effect of developing flow and thermal regime on momentum and heat transfer in micro-scale heat sink. International Journal of Heat and Mass Transfer 2007; 50:3100-3114.

7. Choy DS, Altman PB. Fall of intradiscal pressure with laser ablation. Journal of Clinical Laser Medicine \& Surgery 1995; 13:149-151.

8. Merriam W, Quinnell R, Stockdale $H$, Wills D. The effect of postural changes on the inferred pressures within the nucleus pulposus during lumbar discography. Spine 1984; 9:405-408.

9. Busscher I, Ploegmakers JJ, Verkerke GJ, Veldhuizen AG. Comparative anatomical dimensions of the complete human and porcine spine. European Spine Journal 2010; 19:1104-1114.

10. Petersohn JD, Conquergood LR, Leung $M$. Acute histologic effects and thermal distribution profile of disc biacuplasty using a novel water-cooled bipolar electrode system in an in vivo porcine model. Pain Medicine 2008; 9:26-32.

11. Choy DS. Percutaneous laser disc decompression (PLDD) update: Focus on device and procedure advances. Journal of Clinical Laser Medicine ex Surgery 1993;
11:181-183.

12. Choy D, Case RB, Fielding W, Hughes J, Liebler W, Ascher P. Percutaneous laser nucleolysis of lumbar disks. The New England Journal of Medicine 1987; 317:771-772.

13. Doita M, Kanatani T, Ozaki T, Matsui $N$, Kurosaka M, Yoshiya S. Influence of macrophage infiltration of herniated disc tissue on the production of matrix metalloproteinases leading to disc resorption. Spine 2001; 26:1522-1527.

14. Woertgen C, Rothoerl RD, Brawanski A. Influence of macrophage infiltration of herniated lumbar disc tissue on outcome after lumbar disc surgery. Spine 2000; 25:871-875.

15. Reinhard S, Kraemer J. Chemonucleolysis versus laser disc decompression: A prospective randomized trial. J Bone Joint Surg Br 1997; 79:246-247.

16. Richter EO, Abramova MV, Cantu F, DeAndres J, Lierz P, Manchiaro P, Buyten JP, Kim JD, Jang JH, Jung $\mathrm{GH}$. Anterior epiduroscopic neural decompression: EightDcenter experience in 154 patients. European Journal of Pain Supplements 2011; 5:401-407. 
